\title{
Effect of different healing abutment materials on parameters of peri-implant infections
}

\author{
K Theisen ${ }^{1}$, B Arefnia ${ }^{1}$, M Koller $^{1}$, M Lorenzoni $^{1}$ \\ ${ }^{1}$ Department of Dentistry and Maxillofacial Surgery, Division of Preventive \\ and Operative Dentistry, Endodontics, Pedodontics and Minimally \\ Invasive Dent, Medical University Graz, Graz, Austria
}

\section{Abstract}

\section{Results}

Research is increasingly being conducted on polymethylmethacrylate (PMMA) as a material for fixed dentures and/or healing abutments. In this prospective study, the effects of different healing abutment materials ( $\mathrm{Ti}, \mathrm{Zr}$, PMMA) on parameters of peri-implant infections were investigated.

30 subjects with two-piece $\mathrm{Ti}$ implants received healing abutments made of $\mathrm{Ti}, \mathrm{Zr}$ or PMMA. Subsequently, different parameters of peri-implant infections were assessed at placement of healing abutments and follow-ups (after 2 weeks, 2, 3, 6, 12, and 15 months):

- Inflammatory response mediated by aMMP-8 level in periimplant sulcus fluid

- Clinical and radiographic periodontal status: pocket probing depths (PPD), bleeding on probing (BOP), plaque index (PI), marginal bone loss (MBL)

No significant differences for preliminary results in aMMP-8 level and in clinical parameters (PPD, BOP, PI, MBL) between the three groups were found. Compared to other studies (e.g. [1], [2]) no signs of peri-implant diseases were observed.
Assessment at baseline (placement of healing abutments) and follow-ups (after 2 weeks, 2, 3, 6, 12, and 15 months) revealed no significant differences in aMMP-8 level and in clinical parameters (PPD, BOP, PI, MBL) between the three groups.

Preliminary aMMP-8 levels showed that Ti had the highest mean values at each examination date followed by PMMA and $\mathrm{Zr}$. All mean values were below $10 \mathrm{ng} / \mathrm{ml}$, whereas values below $8 \mathrm{ng} / \mathrm{ml}$ are considered as healthy in most standardized aMMP8-tests (e.g. dentoELISA aMMP-8, dentognostics $\mathrm{GmbH}$, Jena) .

Preliminary 12-months results of PPD, BOP, and PI are displayed. Compared to other studies (e.g. [1], [2]) these values represent no signs of peri-implant diseases.
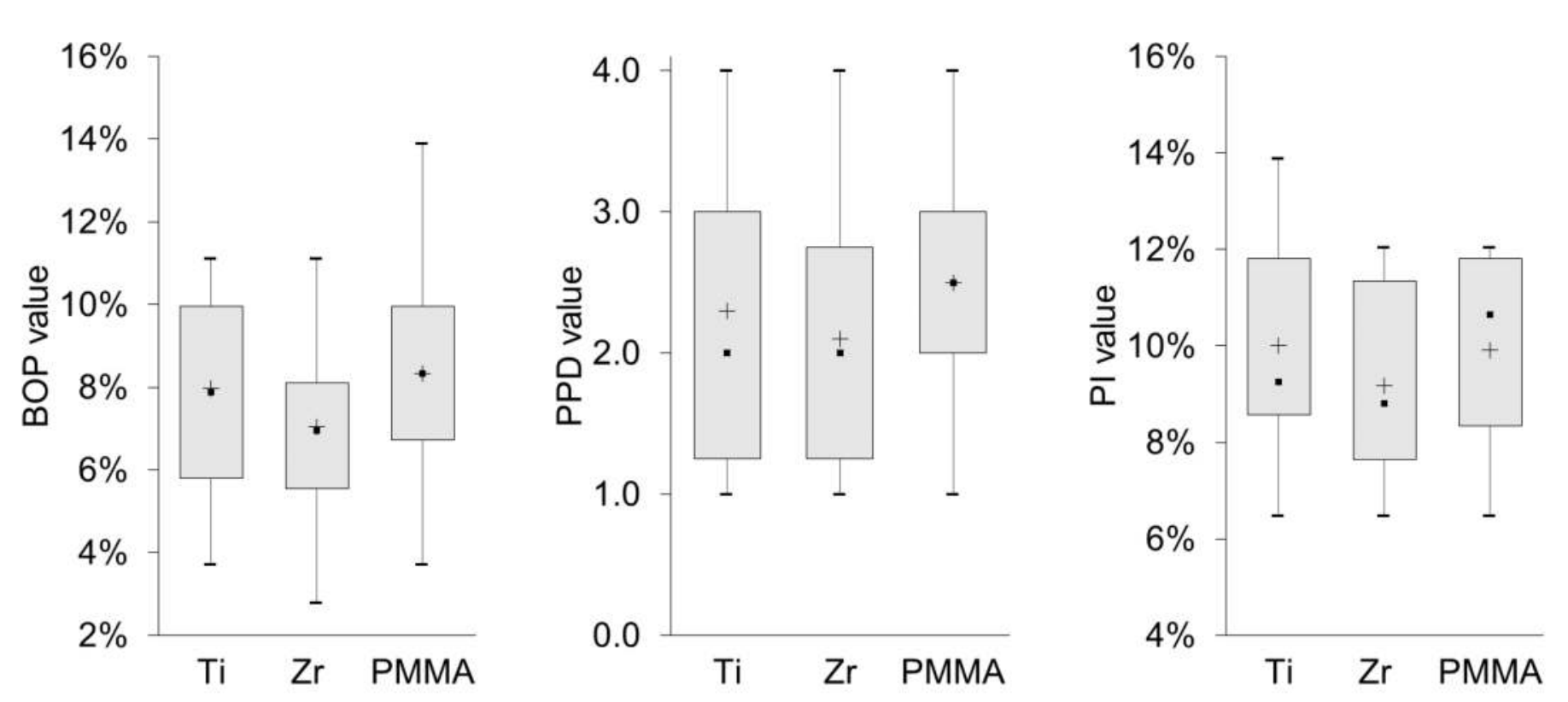

\section{Background and Aim}

Besides $\mathrm{Zr}$ - and Ti-based materials, research is increasingly being conducted on PMMA as a material for fixed dentures and/or healing abutments. The effects of healing abutments with PMMA surfaces on parameters of peri-implant infections have not been investigated.

Aim of this prospective study was to compare the effect of different healing abutment materials ( $\mathrm{Ti}, \mathrm{Zr}$, PMMA) on parameters of periimplant infections. The hypothesis was that there is no difference in clinical and histological parameters.

\section{Conclusion}

The presented results showed that clinical parameters were comparable to the results of other publications. Thus, all materials seem appropriate as healing abutments concerning biological complications.

Further long-term investigations are necessary prior to application of PMMA as material for abutments and permanent prosthetic restorations.

\section{Methods and Materials}

30 subjects with two-piece $\mathrm{Ti}$ implants received healing abutments made of $\mathrm{Ti}, \mathrm{Zr}$ or PMMA. 6 months after surgery, prosthetic restoration was performed (Ti, Zr: all-ceramic; PMMA: PMMA restoration). The following parameters were evaluated:

- Inflammatory response mediated by aMMP-8 level in periimplant sulcus fluid

- Parameters of peri-implant infections: PPD, BOP, PI, MBL

\section{References}

[1] Linkevicius, T. \& Vaitelis, J. 2015, "The effect of zirconia or titanium as abutment material on soft peri-implant tissues: a systematic review and metaanalysis", Clinical oral implants research, vol. 26 Suppl 11, pp. 139-147. [2] Zembic, A., Bosch, A., Jung, R.E., Hammerle, C.H. \& Sailer, I. 2013, "Fiveyear results of a randomized controlled clinical trial comparing zirconia and titanium abutments supporting single-implant crowns in canine and posterior regions", Clinical oral implants research, vol. 24, no. 4, pp. 384-390. 